\section{GP142 LEVEL OF 25(OH)-VITAMIN D AND VITAMIN D RECEPTOR (VDR) GENE POLYMORPHISMS IN ADOLESCENTS WITH CHRONIC GASTRODUODENITIS}

Olga Guzeeva', 'Irina Melnikova, 'Valentina Larionova, ${ }^{2}$ Valeria Novikova*, ${ }^{2}$ Diana Kuzmina. 'North-Western State Medical University named after Mechnikov, St. Petersburg, Russian Federation; ${ }^{2}$ St. Petersburg State Pediatric Medical University, St. Petersburg, Russian Federation

\subsection{6/archdischild-2019-epa.206}

Introduction Chronic gastroduodenitis in adolescents is often accompanied by a decrease in bone density. The mechanisms of this relationship are not fully understood.

Objective To study the level of $25(\mathrm{OH})$-vitamin D, vitamin D receptor gene polymorphisms and bone metabolism in adolescents with chronic gastroduodenitis in dependency of helicobacter pylori (HP) infection.

Materials and methods 51 adolescents aged from 12 to 15 years old were examined. All patients had a morphologically checked chronic gastroduodenitis condition.

Group 1 included 19 patients with HP infection and other 32 children without HP infection were put in a group 2. All children had a gastroenterological examination including urease test and taking a biopsy sample from the stomach. 25 $(\mathrm{OH})$-vitamin $\mathrm{D}$ in serum was determined by ELISA. Polymorphisms (ApaI, TagI, BsmI) of vitamin D receptor gene (VDR) were determined by PCR with restriction digest. Dual-energy $\mathrm{x}$-ray absorptiometry of the lumbar region of the spine L1-L4 was carried out in all patients on a Hologic QDR 4500C densitometer equipped with pediatric reference base in order to study osteocalcin $(\mathrm{OK})$ and $\beta$-Cross-Laps.

Results A $25(\mathrm{OH})$-vitamin D deficiency was observed with an equal frequency in both groups $(5.26 \%$ and $6.25 \%, \mathrm{p}>0.05)$. The correlation between vitamin $\mathrm{D}$ levels and the presence of HP- infection was not found ( $\mathrm{s}=0.417, \mathrm{p} \& \mathrm{gt}$; 0.05). At the same time, the frequency of occurrence of genotypes containing the allele B of the VDR BsmI gene was higher among patients without HP infection compared with children with Hp infection ( $\mathrm{p} \& \mathrm{lt} ; 0.05 \chi 2=6.03$ ). In both groups, the carriers of the VDR BsmI gene $\mathrm{B}$ had a significantly higher level of osteocalcin in comparison with children without it $(90.43 \pm 36.03 \mathrm{ng} / \mathrm{ml}$ and63.02 $\pm 34.12 \mathrm{ng} / \mathrm{ml}, \mathrm{p}<0.005)$. The carriers of the Apal gene A allele in both groups had high bone mineral density and high levels of $25(\mathrm{OH})$-vitamin $\mathrm{D}$, osteocalcin, and $\beta$-cross-laps in comparison to children without this allele. Children with hp-infection, carriers of the $t+$ genotype of the TagI gene of the VDR gene had a higher bone mineral density $(\mathrm{p}=0.001)$.

Conclusion In adolescents with chronic gastroduodenitis, the bone metabolism and the level of $25(\mathrm{OH})$-vitamin $\mathrm{D}$ are related to genetic polymorphism gene VDR, and the presence of HP- infection as well.

\section{GP143 COELIAC DISEASE AND IGA DEFICIENCY IN PAEDIATRIC DIABETES - HOW OFTEN SHOULD WE BE SCREENING OUR PATIENTS?}

${ }^{1}$ Fiona O'Riordan*, ${ }^{1,2}$ Edna Roche, ${ }^{1}$ Ciara McDonnell. 'National Children's Hospital, Tallaght University Hospital, Dublin, Ireland; ${ }^{2}$ Trinity College Dublin, The University of Dublin, Dublin, Ireland

10.1136/archdischild-2019-epa.207

Aims Coeliac disease [CD] and IgA deficiency are more common in patients with Type 1 Diabetes Mellitus [T1D] than in the general population. Optimal screening for these conditions varies worldwide. Current guidance from the International Society for Paediatric and Adolescent Diabetes [ISPAD] is to screen at T1D diagnosis and every 1-2 years thereafter. As IgA deficiency can cause a false negative, CD screening includes measuring IgA Anti-Tissue Transglutaminase antibodies [TTG], serum IgA, and, with IgA deficiency, IgG TTG antibodies. The first aim of our audit was to compare the current screening practice at our centre against ISPAD guidance. Our second aim was to evaluate the need to screen for these conditions every 1-2 years by evaluating changes in TTG and IgA levels following T1D diagnosis.

Methods The study design is a single center, retrospective study of CD screening carried out on 150 consecutive patients attending diabetes services at TUH and diagnosed with T1D over a fifteen year period (2003 - 2018).

Results CD was diagnosed in 15 patients (10\%). TTG was tested at T1D diagnosis in 98 patients $(65.3 \%)$ and 1-2 yearly in 88 patients $(58.7 \%)$. Four patients $(2.7 \%)$ with initially negative TTG results subsequently developed elevated TTG levels and were diagnosed with $\mathrm{CD}$. IgA was measured at diagnosis in 83 patients $(55.3 \%)$ and $1-2$ yearly in 44 patients $(29.3 \%)$. Ten patients $(6.7 \%)$ have not received IgA testing to date. Two patients $(1.3 \%)$ were diagnosed with IgA deficiency on first IgA testing. Of the 138 patients with normal first IgA levels (92\%), none have developed IgA deficiency during a mean 5.5 years of follow-up testing.

Conclusion Our study shows a lack of consistency in CD screening. On the basis of this study, we have initiated a clear CD screening protocol to be used at diabetes out-patient clinics. The results support continuing to monitor TTG levels every 1-2 years to detect changes in TTG levels as four patients with initially negative $\mathrm{CD}$ screening subsequently developed positive TTG and IgA EMA results. They also support measuring IgA levels at diagnosis of T1D to detect IgA deficiency. The results do not provide evidence for continuing to monitor IgA levels every 1-2 years as the IgA status of those with normal IgA levels at T1D diagnosis did not change. We believe a multi-centre prospective study is warranted to further evaluate the need to continue to screen for IgA deficiency after T1D diagnosis.

\section{GP144 QUALITY OF LIFE AND THE IMPACT OF CARING FOR A CHILD WITH PRADER WILLI SYNDROME}

${ }^{1}$ Christina Meade*, ${ }^{1}$ Ruth Martin, ${ }^{2}$ Jacqueline Lyons, ${ }^{2}$ Ann McCrann, ${ }^{3,2}{ }^{2}$ Edna Roche. ${ }^{1}$ Department of Nutrition and Dietetics, Tallaght University Hospital, Tallaght, Dublin, Ireland; ' ${ }^{2}$ Department of Endocrinology and Diabetes, Tallaght University Hospital, Tallaght, Dublin, Ireland; ${ }^{3}$ Department of Paediatrics, Trinity College, The University of Dublin, Dublin, Ireland

\subsection{6/archdischild-2019-epa.208}

Aims To evaluate the family impact of caring for a child with Prader Willi Syndrome (PWS), the caregiver burden and the parents perceived impact on the affected child's Quality of Life (QOL).

Methods All children diagnosed with PWS, attending the service were invited to participate $(n=44)$. QOL was evaluated using the PedsQL questionnaire. The Family Impact module was used to measure the impact of PWS on the family unit. Parent Proxy Reports were used to evaluate the parent's perception of the child's QOL. The PWS Burden questionnaire was used to assess additional challenges associated with managing a child with PWS. 
Results Participants included nineteen children. Median age was 7.9 years (range 0.6 years - 18.1 years). Majority were female $(n=14,74 \%)$. Median age at diagnosis was 2.5 weeks (range birth - 2.7 years). Growth Hormone treatment was in place for the majority $(n=14,74 \%)$ commenced at median age of 2.6 years. Of the reporting parents, $89 \%(n=17)$ were mothers, $37 \%(n=7)$ reported to be homemakers. All children were living in 2 parent households.

QOL family impact Families reported 'Worry' about medical treatment, the future, others reactions to their child's condition and impact on other family members as impacting most significantly on the family.

Significant negative correlations between family QOL and age/weight were observed across many domains, the strongest apparent in the area of family functioning and family relationships.

QOL parent proxy reports PWS was perceived by parents to impact most significantly on their child's School functioning. Significant negative correlations with age and weight were observed. Parents identified psychosocial health and social functioning to be most notably affected.

Burden Various areas were identified as significantly adding to caregiver burden such as concerns regarding long-term health and disruptions to family routines.

Conclusion PWS impacts significantly on QOL for the affected child and the entire family.

\section{GP145 TEN MINUTE TALKS - A NOVEL APPROACH TO DELIVERING TEACHING IN A BUSY MEDICAL UNIT}

${ }^{1}$ Jennifer Boyle ${ }^{*},{ }^{2}$ Dominic Cochran, ${ }^{2}$ Neil Patel. ${ }^{1}$ Neonatal unit, Royal Hospital for Children, Glasgow, Glasgow, UK; ${ }^{2}$ Neonatal Unit, Royal Hospital for Children, Glasgow, Glasgow, UK

10.1136/archdischild-2019-epa.209

Introduction It can be challenging to deliver teaching for trainees in busy medical units. One of us (NP) came up with the idea of brief, ten minute teaching sessions following morning handover. The aim was to achieve focused teaching for day and night shift staff in a brief period without impacting upon clinical duties.

Methods Ten minute talks (TMTs) are delivered by consultants on topics of their choosing after morning handover three days a week. The speaker simply talks to the group, usually without visual aids. A kitchen timer is used to alert the group when ten minutes is up ensuring night staff get away promptly and day staff are free to start their duties. Feedback was collated from trainees (anonymously) and consultants to assess success of this new method.

Results/Discussion The development of TMTs has been well received. A wide range of topics have been covered including clinical subjects, items relating to governance and to practical procedures. Trainees have rated TMTs as their favourite method of teaching when compared to more traditional methods such as grand round, journal club and structured 1 hour teaching presentations. On a ten-point scale (1 poor, 10 excellent) they rated TMTs a mean score of 9.7 compared to our other educational activities, which scored $5,8.3$, \& 8 respectively. Similarly, consultants gave a positive review of TMTs rating them 8.5 in terms of effective teaching.

Conclusion We believe TMTs have proved a popular and effective addition to the education programme in our unit and have ensured that focused relevant teaching is delivered thrice weekly to both day and night shift staff in a time pressured environment with a minimum of resources or preparation. We believe this format could easily be adapted to be used in other medical departments to complement their existing teaching.

\section{GP146 THE DEVELOPMENT, IMPLEMENTATION AND EVALUATION OF AN INNOVATIVE NEAR-PEER MENTORING PROGRAMME FOR DOCTORS IN A TERTIARY NEONATAL UNIT}

Bronwyn Power*, Anna Curley. The National Maternity Hospital, Holles Street, Dublin, Ireland

\subsection{6/archdischild-2019-epa.210}

Introduction Near-Peer Mentoring is a validated educational model for personal and professional development ${ }^{1}$. The European Working Time Directive has resulted in reduced, fragmented working hours for Non-Consultant Hospital Doctors (NCHDs) in Ireland. As a result, sustained professional relationships with experienced colleagues may be harder to establish, and mentoring relationships less likely to develop. We aimed to develop and implement a NearPeer Mentoring Programme for NCHDs in a tertiary neonatal unit.

Methods This was a prospective, questionnaire-based, quality improvement initiative. An Education Session on Mentoring was held for all NCHDs working within the unit. Prospective Mentors were asked to meet formally with their Mentees at least four times over a 3-month period. A Mentoring Template was developed to provide a framework for both the Mentors (Registrars/Specialist Registrars) and Mentees (Senior House Officers). This Template was based on four key themes - Paediatric Training in Ireland, Career Development, Professional Skills and Work-Life Balance. Validated, anonymous questionnaires were distributed prior to and following the period of mentorship.

Results All NCHDs wished to be included in the programme, a total of 9 Mentors and 9 Mentees. There was a $100 \%$ response rate to the questionnaires. Three Mentors (33\%) had received prior mentoring training. Prior to participation, eight Mentors (89\%) felt at least moderately skilled in each of the core mentoring skills outlined in the validated Mentor Competency Assessment Tool.

Mentors and Mentees met on average three times over the study period. $100 \%$ of the Mentees and eight of the Mentors (89\%) felt that the programme contributed to a more positive work environment for NCHDs. Seven Mentees (78\%) felt that their clinical skills and knowledge had improved, that their exposure to clinical activities in the NICU was increased, and that a supportive environment was developed. Six Mentees $(67 \%)$ noted an improvement in their procedural skills. Eight (89\%) agreed that their Mentor was accessible and supportive, provided appropriate career guidance and motivated them to reach their objectives.

Conclusion Although mentoring is a core skill specified by the Royal College of Physicians in Ireland for Higher Specialist Trainees in Paediatrics, there is a paucity of formal Mentoring Programmes for NCHDs. This study has highlighted the benefits of a successful Near-Peer Mentorship Programme for Paediatric trainees. 